\title{
Indifference pricing with counterparty risk
}

\author{
M. NGO, T. NGUYEN, and T. DUONG* \\ John von Neumann Institute-Vietnam National University, VNU IT Park, Linh Trung Ward, Thu Duc District, HCMC, Vietnam
}

\begin{abstract}
We present counterparty risk by a jump in the underlying price and a structural change of the price process after the default of the counterparty. The default time is modeled by a default-density approach. Then we study an exponential utility-indifference price of an European option whose underlying asset is exposed to this counterparty risk. Utility-indifference pricing method normally consists in solving two optimization problems. However, by using the minimal entropy martingale measure, we reduce it to solving only one optimal control problem. In addition, to overcome the incompleteness obstacle generated by the possible jump and the change in structure of the price process, we employ the BSDE-decomposition approach in order to decompose the problem into a global-before-default optimal control problem and an after-default one. Each problem works in its own complete framework. We demonstrate the result by numerical simulation of an European option price under the impact of the size of the jump, intensity of the default, absolute risk aversion and change in the underlying volatility.
\end{abstract}

Key words: indifference pricing, counterparty risk, minimal entropy, BSDE.

\section{Introduction}

In financial market, a firm's price could be influenced by the default of another counterparty. Generally, this default will induce a drop in the firm's stock value, although sometimes this stock value could rise after a counterparty's default. The drop corresponds to a contagious loss when the asset is positively correlated with the counterparty, while the rise often represents a negative correlation situation. Moreover, the default of a counterparty can increase (or decrease) the volatility of the firm's stock, as observed in the past crisis. In this paper, we study the pricing of an European option whose underlying asset is exposed to a counterparty risk. The global market information is modeled by a progressive enlargement of a reference filtration (see [1]), denoted by $\mathbb{F}$, representing the default-free information, and the dependence of default times $\tau$ is modeled by a conditional density hypothesis. The default time $\tau$ is in general a totally inaccessible stopping time with respect to the enlarged filtration $\mathbb{G}$, but is not $\mathbb{F}$-stopping time. In this incomplete market we use the utility-indifference pricing method first adapted in [2]. The advantage of this method is the inclusion of its economic justification and risk aversion, but the disadvantage is that we have to consider two optimal investment problems with and without trading a derivative.

Our main contribution in this paper is the calculation of the minimal entropy martingale measure (MEMM) for our pricing model which includes a jump and changes in the coefficients of the price process after the default time of the counterparty. By using this MEMM and the result in [3], we can reduce the utility indifference pricing problem to only one optimal control

*e-mail: thanh.duong@jvn.edu.vn

Manuscript submitted 2017-01-11, revised 2017-04-12 and 2017-05-10, initially accepted for publication 2017-05-15, published in October 2017. problem and utilize its advantages for an exponential utility function. We then employ the decomposition of the value function before and after default proposed by [4], which separates the problem into after-default and global before-default subproblems, and solves each subproblem by considering a backward stochastic differential equation (BSDE). We solve the utility-indifference price with exponential utility function for a vanilla option whose underlying asset is influenced by counterparty risk in which the underlying asset experiences not only a jump in price, but also changes in its drift and/or volatility.

The paper is structured as follows. Section 2 lays out the model and the option pricing problem with a default density hypothesis. In Section 3 we present the minimal entropy martingale measure approach (MEMM) to solve the utility-difference pricing problem as well as the resulting MEMM density of our problem. Once we have this MEMM density, the option price is obtained using the decomposition approach and the BSDE calculation in Section 4. Finally we demonstrate the numerical simulation of a basic European option in Section 5.

\section{Basic definition and hypothesis}

In our model, the risky asset subject to a counterparty risk is denoted by a stochastic process $S=\left(S_{t}\right)_{t \geq 0}$. Our objective is to calculate the price of an European derivative (option) mature at a finite time horizon $T$ on this security.

We consider a probability space $(\Omega, \mathscr{F}, \mathbb{P})$ equipped with a Brownian motion $W=\left(W_{t}\right), t \in[0, T]$ over a finite time horizon $T<\infty$ and its natural filtration $\mathbb{F}=\left(\mathscr{F}_{t}\right), t \in[0, T]$ satisfying the usual conditions of right-continuity and completeness. The default time is defined by a non-negative and finite random variable $\tau$ on $(\Omega, \mathscr{G}, \mathbb{P})$. Before the default time $\tau$, filtration $\mathbb{F}$ represents the information accessible to the investors. When the default occurs, the investors observe it and add this 
new information $\tau$ to the reference filtration $\mathbb{F}$. We then introduce the jump process $D_{t}=I_{\tau<t}, 0 \leq t \leq T$, and $\mathbb{D}=\left(\mathscr{D}_{t}\right)$, $t \in[0, T]$ is the filtration generated by this jump process. Finally, enlarged progressive filtration $\mathbb{F} \vee \mathbb{D}$, denoted by $\mathbb{G}=\left(\mathscr{G}_{t}\right)$, $t \in[0, T]$ represents the global information available for the investors over $[0, T]$.

In the sequel, we make the basic assumption on the default time of the counterparty, called the density hypothesis (see [1]).

Hypothesis 1. DH. For any $t \in[0, T]$, the conditional distribution of $\tau$ given $\mathscr{F}_{t}$ admits a density with respect to the Lebesgue measure, i.e. there exists a family of $\mathscr{F}_{t} \otimes \mathscr{B}\left(\mathscr{R}_{+}\right)$-measurable positive functions $(\omega, \theta) \rightarrow \alpha_{t}(\theta)$ such that

$$
\mathbb{P}\left[\tau \in d \theta \mid \mathscr{F}_{t}\right]=\alpha_{t}(\theta) d \theta
$$

We note that for any $\theta \geq 0$, the process $\left\{\alpha_{t}(\theta), 0 \leq t \leq T\right\}$ is a $(\mathbb{P}, \mathbb{F})$-martingale.

Under the hypothesis $(\mathbf{D H})$, the $(\mathbb{P}, \mathbb{F})$-Brownian motion $W$ is a $\mathbb{G}$-semimartingale and admits an explicit decomposition in terms of the density $\alpha$ given by (see $[1,5,6]$ )

$$
W_{t}^{\mathbb{G}}=W_{t}-\int_{0}^{t} J_{s} d s, \quad 0 \leq t \leq T,
$$

where $W^{\mathbb{G}}$ is a $(\mathbb{P}, \mathbb{G})$-Brownian motion and $\int_{0}^{t} J_{s} d s$ is a finite variation $\mathbb{G}$-adapted process defined by

$$
\int_{0}^{t} J_{s} d s=\int_{0}^{t \wedge \tau} \frac{d\langle W, G\rangle_{s}}{G_{s}}+\int_{\tau}^{t} \frac{d\langle W, \alpha(\tau)\rangle_{s}}{\alpha_{s}(\tau)}, \quad 0 \leq t \leq T,
$$

where $G_{t}=\mathbb{P}\left[\tau>t \mid \mathscr{F}_{t}\right]$ is the conditional survival probability. $J$ admits the following decomposition

$$
J_{t}=J_{t}^{\mathbb{F}} I_{\tau>t}+J_{t}^{d}(\tau) I_{\tau \leq t}, \quad 0 \leq t \leq T
$$

Furthermore, the process

$$
M_{t}=D_{t}-\int_{0}^{t} A_{s} d s, \quad 0 \leq t \leq T,
$$

is a $(\mathbb{P}, \mathbb{G})$-martingale (see [1]), where

$$
\int_{0}^{t} A_{s} d s=\int_{0}^{\tau \wedge t} \frac{\alpha_{\theta}(\theta)}{G_{\theta}} d \theta, \quad 0 \leq t \leq T
$$

is the $(\mathbb{P}, \mathbb{G})$-predictable compensator of jump process $D$. By denoting $\lambda_{t}=\frac{\alpha_{t}(t)}{G_{t}}$, we have

$$
A_{t}=\lambda_{t}\left(1-D_{t}\right), \quad 0 \leq t \leq T
$$

\section{- Asset model:}

The dynamics of the risky asset subject to a counterparty risk is represented by a $\mathbb{G}$-adapted discounted price process such that

$$
S_{t}=S_{t}^{\mathbb{F}} I_{\tau>t}+S_{t}^{d}(\tau) I_{\tau \leq t}, \quad t \in[0, T],
$$

where $S^{\mathbb{F}}$ is an $\mathbb{F}$-adapted process representing the discounted price process in the default-free market, governed by:

$$
\begin{aligned}
d S_{t}^{\mathbb{F}} & =S_{t}^{\mathbb{F}}\left(\mu_{t}^{\mathbb{F}} d t+\sigma_{t}^{\mathbb{F}} d W_{t}\right) \\
S_{0}^{\mathbb{F}} & =S_{0^{-}}, \quad 0 \leq t \leq T
\end{aligned}
$$

and $\left\{S_{t}^{d}(\theta), \theta \leq t \leq T, \theta \in[0, T]\right\}$ is a measurable (in $\theta$ ) family of $\mathbb{F}$-adapted processes representing the discounted price process after the default at time $\tau=\theta$, governed by

$$
\begin{aligned}
d S_{t}^{d}(\theta) & =S_{t}^{d}(\theta)\left(\mu_{t}^{d}(\theta) d t+\sigma_{t}^{d}(\theta) d W_{t}\right), \\
S_{\theta}^{d}(\theta) & =S_{\theta^{-}}^{\mathbb{F}}\left(1+\gamma_{\theta}\right), \quad \theta<t \leq T,
\end{aligned}
$$

where $\mu^{\mathbb{F}}, \sigma^{\mathbb{F}}$ are $\mathbb{F}$-adapted processes and $\mu_{t}^{d}(\theta), \sigma_{t}^{d}(\theta)$ are $\mathscr{F}_{t} \otimes \mathscr{B}\left(\mathbb{R}^{+}\right)$measurable functions for all $t \in[0, T]$ and $\gamma$ is $\mathbb{F}$-adapted process that represents the percentage price's change immediately at the default time of the counterparty. We assume that for all $t \in[0, T], \sigma_{t}>0$ and $\gamma_{t} \in(-1,1)$ almost everywhere, and the following integrability condition is satisfied for all $\theta \in[0, T]$

$$
\begin{aligned}
& \int_{0}^{T}\left(\frac{\mu_{t}^{\mathbb{F}}}{\sigma_{t}^{\mathbb{F}}}\right)^{2} d t+\int_{\theta}^{T}\left(\frac{\mu_{t}^{d}(\theta)}{\sigma_{t}^{d}(\theta)}\right)^{2} d t \\
& +\int_{0}^{T}\left(\sigma_{t}^{\mathbb{F}}\right)^{2} d t+\int_{\theta}^{T}\left(\sigma_{t}^{d}(\theta)\right)^{2} d t<\infty, \quad \text { a.s }
\end{aligned}
$$

which ensure that the dynamics of the discounted price process is well defined.

We denote two $\mathbb{G}$-adapted processes $\mu$ and $\sigma$ by

$$
\begin{aligned}
& \mu_{t}=\mu_{t}^{\mathbb{F}} I_{\tau>t}+\mu_{t}^{d}(\tau) I_{\tau \leq t}, \\
& \sigma_{t}=\sigma_{t}^{\mathbb{F}} I_{\tau>t}+\sigma_{t}^{d}(\tau) I_{\tau \leq t} .
\end{aligned}
$$

We can see from $(4,5)$ and $(6)$ that the dynamics of the discounted stock price process $S$ can be written as:

$$
d S_{t}=S_{t^{-}}\left(\mu_{t} d t+\sigma_{t} d W_{t}+\gamma_{t} d D_{t}\right), \quad 0 \leq t \leq T .
$$

The interpretation of the contagion risk model for the discounted asset price $S$ is as follows. The process $S^{\mathbb{F}}$ represents the asset price before the default, and there is a jump in stock price at the default time of the counterparty, whose size is represented by the process $\gamma$, which may take positive or negative values, corresponding to the proportional gain or loss on the stock price. After the default at time $\tau=\theta, S^{d}(\theta)$ represents the asset price process, where there is a change in the coefficients depending on the default time, for example, if we expect that the volatility $\sigma^{d}(\theta)$ after default is greater than the volatility $\sigma^{\mathbb{F}}$ before default, we can specify $\sigma^{d}(\theta)$ to be of the form $\sigma_{t}^{d}(\theta)=\sigma$ $\mathbb{F}+a e^{-b(t-\theta)}, a>0$.

\section{- Wealth dynamic:}

Let $\bar{\pi}=\left(\bar{\pi}_{t}\right)_{t \in[0, T]}$, which is $\mathbb{G}$-predictable, denote the amount of wealth invested at time $t$ in the stock (also called trading strategy). We also define the discounted strategy process $\pi_{t}=e^{-r t} \bar{\pi}_{t}$, 
where $r$ is the risk-free rate. Similarly to the previous section, $\bar{\pi}$ could be decomposed into the form

$$
\bar{\pi}_{t}=\bar{\pi}_{t}^{\mathbb{F}} I_{\tau \geq t}+\bar{\pi}_{t}^{d}(\tau) I_{\tau<t}, \quad 0 \leq t \leq T .
$$

The investor's wealth, decomposed as

$$
\bar{X}_{t}^{\bar{\pi}}=\bar{X}_{t}^{\bar{\pi}, \mathbb{F}} I_{\tau>t}+\bar{X}_{t}^{\bar{\pi}, d}(\tau) I_{\tau \leq t}, \quad 0 \leq t \leq T,
$$

is a $\mathbb{G}$-adapted process following the dynamics

$$
d \bar{X}_{t}^{\bar{\pi}}=\bar{\pi}_{t} \frac{d \bar{S}_{t}}{\bar{S}_{t^{-}}}+\left(\bar{X}_{t}^{\bar{\pi}}-\bar{\pi}_{t}\right) r d t, \quad 0 \leq t \leq T,
$$

where $\bar{S}_{t}=e^{r t} S_{t}$ is the stock price process.

Finally, the discounted wealth process $X_{t}^{\pi}=e^{-r t} \bar{X}_{t}^{\bar{\pi}}$ has the following dynamics

$$
d X_{t}^{\pi}=\bar{\pi}_{t} e^{-r t} \frac{d S_{t}}{S_{t^{-}}}=\pi_{t} \frac{d S_{t}}{S_{t^{-}}}, \quad 0 \leq t \leq T,
$$

and decomposition

$$
\begin{aligned}
X_{t}^{\pi} & =X_{t}^{\pi^{\mathbb{F}}, \mathbb{F}} I_{\tau>t}+X_{t}^{\pi^{d}(\tau), d}(\tau) I_{\tau \leq t}, \quad 0 \leq t \leq T, \\
\pi_{t} & =\pi_{t}^{\mathbb{F}} I_{\tau \geq t}+\pi_{t}^{d}(\tau) I_{\tau<t}, \quad 0 \leq t \leq T,
\end{aligned}
$$

where $X^{\pi^{\mathbb{F}}, \mathbb{F}}$ is the discounted wealth process before default, governed by

$$
\begin{aligned}
d X_{t}^{\pi^{\mathbb{F}}, \mathbb{F}} & =\pi_{t}^{\mathbb{F}} \frac{d S_{t}^{\mathbb{F}}}{S_{t}^{\mathbb{F}}}, \\
X_{0}^{\pi^{\mathbb{F}}, \mathbb{F}} & =X_{0^{-}}, \quad 0 \leq t \leq T,
\end{aligned}
$$

and $X^{\pi^{d}(\theta), d}(\theta)$ is the discounted wealth process after the default takes place at $\tau=\theta$, governed by

$$
\begin{aligned}
d X_{t}^{\pi^{d}(\theta), d}(\theta) & =\pi_{t}^{d}(\theta) \frac{d S_{t}^{d}(\theta)}{S_{t}^{d}(\theta)}, \quad \theta<t \leq T, \\
X_{\theta}^{\pi^{d}(\theta), d}(\theta) & =X_{\theta^{-}}^{\pi, \mathbb{F}}+\pi_{\theta}^{\mathbb{F}} \gamma_{\theta} .
\end{aligned}
$$

\section{Minimal entropy martingale measure and its relation to Indifference pricing method}

We consider the valuation problem of a derivative on the risky asset $S$ by utility indifference pricing method. The maximal expected utility one can obtain by trading in $S$ via some strategy $\pi$, if one starts with $\mathrm{x}$ and has to pay out $B$ at $T$, is $V_{B}(x)=\sup _{\pi \in \mathscr{A}} E\left[U\left(X_{T}^{\pi}-B\right) \mid X_{0}^{\pi}=x\right]$, where $\mathscr{A}$ is the set of admissible trading strategies.

The utility indifference price $\operatorname{Pr}$ of the derivative is implicitly defined by

$$
V_{0}(x)=V_{B}(x+P r)
$$

where $V_{0}(x)=\sup _{\pi \in \mathscr{A}} E\left[U\left(X_{T}^{\pi}\right) \mid X_{0}^{\pi}=x\right]$ is the maximal conditional expected utility we can achieve by starting with initial capital $x$ and do not pay out anything at the maturity $T$. In other words, $V_{0}(x)$ is the value-function of the optimization problem without trading derivative. Utility indifference price $P r$ is the price which equates the expected utility including the contingent claim $B$ with the expected utility without the contingent claim $B$. That is, in this case, the utility indifference price is the minimum price of the contingent claim $B$ for the investor as a seller.

The solution of the indifference pricing equation (9) has been given perfectly for the case of an exponential utility using the minimal entropy martingale measure (see Definition 1 below) in [3]. The authors of [3] reduced the problem of solving two optimizations in both sides of equation (9) to a single one, then associated this problem with the finding of a minimal entropy martingale measure, of which the definition is given below.

Definition 1. Denote

$$
\begin{aligned}
P_{e} & =\{Q \sim \mathbb{P} \mid \mathrm{S} \text { is local }(Q, \mathbb{G}) \text {-martingale }\}, \\
P_{e, f} & =\left\{Q \in P_{e} \mid H(Q \mid \mathbb{P})<\infty\right\},
\end{aligned}
$$

where $H(Q \mid \mathbb{P})$ is the relative entropy of $Q$ with respect to $\mathbb{P}$, i.e.

$$
H(Q \mid \mathbb{P})= \begin{cases}\int_{\Omega} \log \frac{d Q}{d \mathbb{P}} d Q, & \text { if } Q<<\mathbb{P} \\ \infty, & \text { otherwise }\end{cases}
$$

If an equivalent local martingale measure $\mathbb{P}^{*} \in P_{e, f}$ satisfy the following condition

$$
H(Q \mid \mathbb{P}) \geq H\left(\mathbb{P}^{*} \mid \mathbb{P}\right), \quad \forall Q \in P_{e, f},
$$

then $\mathbb{P}^{*}$ is called a MEMM.

According to Proposition 3 in [3], in case of an exponential utility,

$$
U(x)=-\exp (-p x), \quad p>0,
$$

the utility indifference price $P r$ of the derivative can be derived as

$$
\operatorname{Pr}=x+\frac{1}{p} \log \left(-\sup _{\pi \in \mathscr{A}} E^{\mathbb{P}^{*}}\left[U\left(X_{T}^{\pi}-B\right) \mid X_{0}^{\pi}=x\right]\right),
$$

where $\mathbb{P}^{*}$ is the minimal entropy martingale measure.

As a result, the utility indifference pricing valuation refers to solving a unique optimization problem of the form

$$
\sup _{\pi \in \mathscr{A}} E^{\mathbb{P}^{*}}\left[U\left(X_{T}^{\pi}-B\right) \mid X_{0}^{\pi}=x\right] .
$$

We now proceed to calculate the MEMM for the risky asset in the defaultable context presented in the previous Section. 
Firstly, we denote $\mathbb{G}$-adapted process $F^{*}$ as follows

$$
\begin{aligned}
F_{t}^{*}(\beta, \phi) & =\beta_{t} \mu_{t}+\frac{1}{2}\left(\sigma_{t} \beta_{t}\right)^{2}+\beta_{t} \sigma_{t} J_{t}+ \\
& +\left(e^{\beta_{t} \gamma_{t}} e^{\phi_{t}}-1\right) A_{t},
\end{aligned}
$$

for all $t \in[0, T]$, where $\beta$ and $\phi$ are $\mathbb{G}$-predictable processes. Similarly to the previous section, $F^{*}, \beta$ and $\phi$ could be decomposed into the following form

$$
\begin{aligned}
F_{t}^{*}(\beta, \phi) & =F_{t}^{\mathbb{F}}(\beta, \phi) I_{\tau>t}+F^{d}(\tau)(\beta) I_{\tau \leq t}, \\
\beta_{t} & =\beta_{t}^{\mathbb{F}} I_{\tau \geq t}+\beta_{t}^{d}(\tau) I_{\tau<t}, \\
\phi_{t} & =\phi_{t}^{\mathbb{F}} I_{\tau \geq t}+\phi_{t}^{d}(\tau) I_{\tau<t},
\end{aligned}
$$

for all $t \in[0, T]$. From the expression of $F_{t}^{*}(11)$ and $A_{t}(3)$ we have

$$
\begin{aligned}
F_{t}^{\mathbb{F}}(\beta, \phi) & =\beta_{t}^{\mathbb{F}} \mu_{t}^{\mathbb{F}}+\frac{1}{2}\left(\sigma_{t}^{\mathbb{F}} \beta_{t}^{\mathbb{F}}\right)^{2}+ \\
& +\beta_{t}^{\mathbb{F}} \sigma_{t}^{\mathbb{F}} J_{t}^{\mathbb{F}}+\lambda_{t}\left(e^{\beta_{t} \gamma_{t}} e^{\phi_{t}}-1\right),
\end{aligned}
$$

and

$$
\begin{aligned}
F_{t}^{d}(\theta)(\beta) & =\beta_{t}^{d}(\theta) \mu_{t}^{d}(\theta)+\frac{1}{2}\left(\sigma_{t}^{d}(\theta) \beta_{t}^{d}(\theta)\right)^{2}+ \\
& +\beta_{t}^{d}(\theta) \sigma_{t}^{d}(\theta) J_{t}^{d}(\theta) .
\end{aligned}
$$

Theorem 1. In the case $\mu_{t}^{\mathbb{F}}, \sigma_{t}^{\mathbb{F}}, \lambda_{t}, J_{t}^{\mathbb{F}}$ and $\mu_{t}^{d}(\theta), \sigma_{t}^{d}(\theta), J_{t}^{d}(\theta)$, $\gamma_{t}$ are deterministic functions $\forall 0 \leq \theta \leq t \leq T$, if there are $\mathbb{G}$-predictable processes $\beta$ and $\phi$ being solution of

$$
\begin{gathered}
\phi_{t}=\int_{t}^{T}\left(F_{s}^{d}(t)(\beta)-F_{s}^{\mathbb{F}}(\beta, \phi)\right) d s, \\
\mu_{t}+\beta_{t} \sigma_{t}^{2}+\sigma_{t} J_{t}+\gamma_{t} A_{t} e^{\beta_{t} \gamma_{t}} e^{\phi_{t}}=0,
\end{gathered}
$$

$\left(F^{\mathbb{F}}, F^{d}\right.$ are defined in (12) and (13), respectively) and satisfying the condition

$$
\begin{aligned}
& E\left[\operatorname { e x p } \left(\frac{1}{2} \int_{0}^{T}\left(\beta_{s} \sigma_{s}\right)^{2} d s+\right.\right. \\
& \left.\left.\quad+\int_{0}^{T}\left(e^{\beta_{s} \gamma_{s}} e^{\phi_{s}}-1\right)^{2} A_{s} d s\right)\right]<\infty,
\end{aligned}
$$

then the probability measure $\mathbb{P}^{*}$ defined by

$$
\frac{d \mathbb{P}^{*}}{d \mathbb{P}}=L_{T},
$$

where

$$
L_{t}=e^{\left(-\int_{0}^{t} F_{s}^{*}(\beta, \phi) d s+\int_{0}^{t} \phi_{s} d D_{s}\right)} e^{\int_{0}^{t} \beta_{s} \frac{d S_{s}}{S_{S-}}}
$$

$\left(F^{*}\right.$ is defined in (11)) is a MEMM of $S$.
Proof. By the assumption $\mu_{t}^{\mathbb{F}}, \sigma_{t}^{\mathbb{F}}, \lambda_{t}, J_{t}^{\mathbb{F}}$ and $\mu_{t}^{d}(\theta), \sigma_{t}^{d}(\theta), J_{t}^{d}(\theta), \gamma_{t}$ are deterministic functions $\forall 0 \leq \theta \leq t \leq T$, and combining (12-14), and (15) we can see that $\phi$ is a $\mathbb{G}$-predictable process (actually, a deterministic function).

From (1) and (2), $W_{t}^{\mathbb{G}}=W_{t}-\int_{0}^{t} J_{s} d s$ is a $(\mathbb{P}, \mathbb{G})$-Brownian motion, and $M_{t}=D_{t}-\int_{0}^{t} A_{s} d s$ is a $(\mathbb{P}, \mathbb{G})$-martingale. For the sake of convenience we define $K_{t}=e^{\int_{0}^{t} \beta_{s} \frac{d S_{s}}{S_{s^{-}}}}$and $H_{t}=$ $=e^{\left(-\int_{0}^{t} F_{s}^{*}(\beta, \phi) d s+\int_{0}^{t} \phi_{s} d D_{s}\right)}$, so that $L_{t}=K_{t} H_{t}$. Applying Ito formula for $K_{t}$ and $H_{t}$ on $[0, T]$, we have, respectively,

$$
\begin{aligned}
d K_{t}= & K_{t^{-}}\left\{\beta_{t} \mu_{t} d t+\beta_{t} \sigma_{t} d W_{t}+\frac{1}{2}\left(\beta_{t} \sigma_{t}\right)^{2} d t+\left(e^{\beta_{t} \gamma_{t}}-1\right) d D_{t}\right\} \\
= & K_{t^{-}}\left\{\beta_{t} \mu_{t}+\frac{1}{2}\left(\sigma_{t} \beta_{t}\right)^{2}+\left(e^{\beta_{t} \gamma_{t}}-1\right) A_{t}+\beta_{t} \sigma_{t} J_{t}\right\} d t \\
& +K_{t^{-}}\left\{\beta_{t} \sigma_{t} d W_{t}^{\mathbb{G}}+\left(e^{\beta_{t} \gamma_{t}}-1\right) d M_{t}\right\},
\end{aligned}
$$

and

$$
\begin{aligned}
d H_{t} & =H_{t^{-}}\left(-F_{t}^{*} d t+\left(e^{\phi_{t}}-1\right) d D_{t}\right) \\
& =H_{t^{-}}\left(-F_{t}^{*} d t+\left(e^{\phi_{t}}-1\right) d M_{t}+\left(e^{\phi_{t}}-1\right) A_{t} d t\right) .
\end{aligned}
$$

Moreover,

$$
\begin{aligned}
d[K, H]_{t} & =K_{t^{-}} H_{t^{-}}\left[\left(e^{\phi_{t}}-1\right)\left(e^{\beta_{t} \gamma_{t}}-1\right) d M_{t}+\right. \\
& \left.+\left(e^{\phi_{t}}-1\right)\left(e^{\beta_{t} \gamma_{t}}-1\right) A_{t} d t\right] .
\end{aligned}
$$

Thus, Ito formula and the definition of $F^{*}(\beta, \phi)(11)$ give the dynamic of the process $L$ as

$$
\begin{aligned}
d L_{t} & =L_{t^{-}}\left\{\left[\left(e^{\phi_{t}}-1\right)+\left(e^{\beta_{t} \gamma_{t}}-1\right)+\left(e^{\phi_{t}}-1\right)\left(e^{\beta_{t} \gamma_{t}}-1\right)\right] d M_{t}\right. \\
& +\beta_{t} \sigma_{t} d W_{t}^{\mathbb{G}}+\left[\beta_{t} \mu_{t}+\frac{1}{2}\left(\sigma_{t} \beta_{t}\right)^{2}+\left(e^{\beta_{t} \gamma_{t}}-1\right) A_{t}+\beta_{t} \sigma_{t} J_{t}\right. \\
& \left.\left.+\left(e^{\phi_{t}}-1\right) A_{t}+\left(e^{\phi_{t}}-1\right)\left(e^{\beta_{t} \gamma_{t}}-1\right) A_{t}-F_{t}^{*}(\beta, \phi)\right] d t\right\} \\
& =L_{t^{-}}\left\{\left(e^{\beta_{t} \gamma_{t}} e^{\phi_{t}}-1\right) d M_{t}+\beta_{t} \sigma_{t} d W_{t}^{\mathbb{G}}\right\}, \quad t \in[0, T] .
\end{aligned}
$$

The Novikov condition (16) is sufficient for $L$ to be a $(\mathbb{P}, \mathbb{G})$-martingale on $[0, T]$ (see Theorem 9 of [7]). Therefore, $L$ is a strictly positive $(\mathbb{P}, \mathbb{G})$-martingale on $[0, T]$ and $E\left[L_{T}\right]=E\left[L_{0}\right]=1$. We define the measure $\mathbb{P}^{*}$ by

$$
\frac{d \mathbb{P}^{*}}{d \mathbb{P}}=L_{T} .
$$

Applying Ito formula for $L_{t} S_{t}$ we have for $t \in[0, T]$,

$$
\begin{aligned}
d\left(L_{t} S_{t}\right)= & L_{t^{-}} S_{t^{-}}\left\{\left(\beta_{t}+1\right) \sigma_{t} d W_{t}^{\mathbb{G}}\right. \\
& +\left[\left(e^{\beta_{t} \gamma_{t}} e^{\phi_{t}}-1\right)+\gamma_{t}+\gamma_{t}\left(e^{\beta_{t} \gamma_{t}} e^{\phi_{t}}-1\right)\right] d M_{t} \\
& \left.+\left(\mu_{t}+\sigma_{t} J_{t}+\beta_{t} \sigma_{t}^{2}+\gamma_{t} A_{t} e^{\beta_{t} \gamma_{t}} e^{\phi_{t}}\right) d t\right\} .
\end{aligned}
$$

Since $\beta, \phi$ satisfies (15), it follows that $S$ is a $\left(\mathbb{P}^{*}, \mathbb{G}\right)$-(local) martingale on $[0, T]$. 
From the definition (14) of $\phi$, in the case of $\tau \leq T$ we have

$$
\begin{aligned}
& -\int_{0}^{T} F_{s}^{*}(\beta, \phi) d s+\int_{0}^{T} \phi_{s} d D_{s} \\
= & -\int_{0}^{T} F_{s}^{*}(\beta, \phi) d s+\int_{\tau}^{T} F_{s}^{d}(\tau)(\beta) d s-\int_{\tau}^{T} F_{s}^{\mathbb{F}}(\beta, \phi) d s \\
= & -\int_{0}^{T} F_{s}^{*}(\beta, \phi) d s+\int_{\tau}^{T} F_{s}^{d}(\tau)(\beta) d s \\
& +\int_{0}^{\tau} F_{s}^{\mathbb{F}}(\beta, \phi) d s-\int_{0}^{T} F_{s}^{\mathbb{F}}(\beta, \phi) d s \\
= & -\int_{0}^{T} F_{s}^{\mathbb{F}}(\beta, \phi) d s,
\end{aligned}
$$

and when $\tau>T$, we have

$$
-\int_{0}^{T} F_{s}^{*}(\beta, \phi) d s+\int_{0}^{T} \phi_{s} d D_{s}=-\int_{0}^{T} F_{s}^{\mathbb{F}}(\beta, \phi) d s .
$$

Moreover, from Definition 1, we have

$$
\begin{aligned}
H\left(\mathbb{P}^{*} \mid \mathbb{P}\right) & =E^{\mathbb{P}^{*}}\left(\log \frac{d \mathbb{P}^{*}}{d \mathbb{P}}\right) \\
& =E^{\mathbb{P}^{*}}\left(-\int_{0}^{T} F_{s}^{*}(\beta, \phi) d s+\int_{0}^{T} \phi_{s} d D_{s}+\int_{0}^{T} \beta_{s} \frac{d S_{s}}{S_{s^{-}}}\right) \\
& =E^{\mathbb{P}^{*}}\left(-\int_{0}^{T} F_{s}^{*}(\beta, \phi) d s+\int_{0}^{T} \phi_{s} d D_{s}\right) \\
& =E^{\mathbb{P}^{*}}\left(-\int_{0}^{T} F_{s}^{\mathbb{F}}(\beta, \phi) d s\right)=-\int_{0}^{T} F_{s}^{\mathbb{F}}(\beta, \phi) d s,
\end{aligned}
$$

$\left(F^{\mathbb{F}}(\beta, \phi)\right.$ is deterministic because $\mu_{t}^{\mathbb{F}}, \sigma_{t}^{\mathbb{F}}, \lambda_{t}, J_{t}^{\mathbb{F}}$ and $\mu_{t}^{d}(\theta), \sigma_{t}^{d}(\theta)$, $J_{t}^{d}(\theta), \gamma_{t}$ are deterministic functions). For any equivalent martingale measure $Q$ (recalling that $E^{Q}\left(\int_{0}^{T} \beta_{s^{-}} \frac{d S_{s}}{S_{s^{-}}}\right)=0$ ), by similar argument we have

$$
\begin{aligned}
E^{Q}\left(\log \frac{d \mathbb{P}^{*}}{d \mathbb{P}}\right) & =E^{Q}\left(-\int_{0}^{T} F_{s}^{\mathbb{F}} d s\right)= \\
& =-\int_{0}^{T} F_{s}^{\mathbb{F}}(\beta, \phi) d s=H\left(\mathbb{P}^{*} \mid \mathbb{P}\right)
\end{aligned}
$$

In consequence,

$$
\begin{aligned}
H(Q \mid \mathbb{P}) & =H\left(Q \mid \mathbb{P}^{*}\right)+E^{Q}\left(\log \frac{d \mathbb{P}^{*}}{d \mathbb{P}}\right) \\
& =H\left(Q \mid \mathbb{P}^{*}\right)+H\left(\mathbb{P}^{*} \mid \mathbb{P}\right)
\end{aligned}
$$

Because $H\left(Q \mid \mathbb{P}^{*}\right) \geq 0$ (see Theorem 1.4.1 of [8]), $\mathbb{P}^{*}$ is MEMM by definition.

\section{Utility indifference price by MEMM method}

Recalling from (10) that the utility indifference price of a vanilla option is

$$
\operatorname{Pr}=x+\frac{1}{p} \log \left(-\sup _{\pi \in \mathscr{A}} E^{\mathbb{P}^{*}}\left[U\left(X_{T}^{\pi}-B\right) \mid X_{0}^{\pi}=x\right]\right),
$$

the valuation therefore consists in solving the optimization problem $\mathscr{B}_{\mathbb{R}^{+}}$

$$
V_{C}(x)=\sup _{\pi \in \mathscr{A}} J_{0}(x, \pi)
$$

where

$$
\begin{aligned}
J_{0}(x, \pi) & =E^{\mathbb{P}^{*}}\left[U\left(X_{T}^{\pi}-B\right) \mid X_{0}^{\pi}=x\right]= \\
& =E\left[U\left(X_{T}^{\pi}-C\right) \mid X_{0}^{\pi}=x\right], \\
C & =B+\frac{1}{p} \log L_{T},
\end{aligned}
$$

and $B$ is the option's discounted pay-off.

In the following subsections, we will solve the optimization problem in a defaultable context by using the approach proposed by [4].

4.1. Decomposition of the optimal control problem. The whole problem is decomposed into two sub-problems: before and after the default. In this work, the pay-off is subject to change depending on the default's occurrence, which sometimes happens in a credit-related product. By definition of $C$ in (19), $C$ could have the $\mathbb{G}$-decomposition of the form

$$
C=C^{\mathbb{F}} I_{\tau>T}+C^{d}(\tau) I_{\tau \leq T},
$$

where $C^{\mathbb{F}}=B^{\mathbb{F}}+\frac{1}{p} \log L_{T}^{\mathbb{F}}$ is $\mathscr{F}_{T}$-measurable and $C^{d}(\theta)=B^{d}(\theta)$ $+\frac{1}{p} \log L_{T}^{d}(\theta)$ is measurable with respect to $\mathscr{F}_{T} \otimes \mathscr{B}_{\mathbb{R}^{+}}$.

We define the value-function process of the after-default optimization problem as

$$
\begin{aligned}
& V_{\theta}^{d}(x)=\operatorname{esssup}_{\pi^{d}(\theta) \in \mathscr{A}^{d}(\theta)} J_{\theta}^{d}\left(x, \pi^{d}(\theta)\right), \\
& (\theta, x) \in[0, T] \times(0, \infty),
\end{aligned}
$$

where

$$
\begin{aligned}
J_{\theta}^{d}\left(x, \pi^{d}(\theta)\right) & =E\left[U\left(X_{T}^{\pi^{d}(\theta), d}(\theta)-C^{d}(\theta)\right) \alpha_{T}(\theta)\right. \\
& \left.\left.-C^{d}(\theta)\right) \alpha_{T}(\theta) \mid \mathscr{F}_{\theta}, X_{\theta}^{\pi^{d}(\theta), d}(\theta)=x\right],
\end{aligned}
$$

and $\mathscr{A}^{d}(\theta)$ is the set of all admissble strategies after default. By using the backward recursive framework with BSDEs system as in [4] (see equations (3.8) and (3.9)), we have the decomposition of the global optimization problem (18) in the following remark

Remark 1. Assume that $V_{\theta}^{d}(x)<\infty$ a.s. for all $(\theta, x) \in[0, T] \times$ $\times(0, \infty)$, then

$$
\begin{aligned}
V_{C}(x) & =\sup _{\pi^{\mathbb{F}} \in \mathscr{A}} E\left[U\left(X_{T}^{\pi^{\mathbb{F}}, \mathbb{F}}-C^{\mathbb{F}}\right) G_{T}+\right. \\
& \left.+\int_{0}^{T} V_{\theta}^{d}\left(X_{\theta}^{\pi^{\mathbb{F}}, \mathbb{F}}+\pi_{\theta}^{\mathbb{F}} \gamma_{\theta}\right) d \theta \mid X_{0}^{\pi^{\mathbb{F}}, \mathbb{F}}=x\right],
\end{aligned}
$$

where $\mathscr{A}^{\mathbb{F}}$ is the set of all admissible strategies before default. 
4.2. Solution of the optimal investment problem. In our model, we allow the drift and diffusion coefficients after the default $\left(\mu^{d}, \sigma^{d}\right)$ to depend on the default time $\tau$. This assumption makes this optimization problem unable to be solved if one uses the classical dynamic programming approach. In order to solve the problem, we first find the after-default value-function and then the before-default one using a recursive BSDE framework as in [4].

4.2.1. The after-default utility maximization problem. In the case of an exponential utility, the recursive framework in [4, Theorem 4.2, case $k=n]$ helps to find the solution of the after-default value function $V_{\theta}^{d}(x)$ in the following simple form

$$
V_{\theta}^{d}(x)=U\left(x-Y_{\theta}^{d}(\theta)\right)=-\exp \left(-p\left(x-Y_{\theta}^{d}(\theta)\right)\right),
$$

where $\left(Y^{d}, Z^{d}\right)$ is a solution of the BSDE

$$
\begin{aligned}
V_{\theta}^{d}(x) & =C^{d}(\theta)+\frac{1}{p} \log \alpha_{T}(\theta)+ \\
& +\int_{t}^{T} f^{d}\left(s, Z_{s}^{d}, \theta\right) d s-\int_{t}^{T} Z_{s}^{d} d W_{s}, \quad \theta \leq t \leq T,
\end{aligned}
$$

with the driver

$$
\begin{aligned}
f^{d}(t, z, \theta)= & -\frac{\mu_{t}^{d}(\theta)}{\sigma_{t}^{d}(\theta)} z-\frac{1}{2 p}\left(\frac{\mu_{t}^{d}(\theta)}{\sigma_{t}^{d}(\theta)}\right)^{2}+\frac{p}{2} \inf _{\alpha \in \mathscr{A}^{d}(\theta)} \mid z \\
& -\sigma_{t}^{d}(\theta) \alpha+\left.\frac{1}{p} \frac{\mu_{t}^{d}(\theta)}{\sigma_{t}^{d}(\theta)}\right|^{2} .
\end{aligned}
$$

4.2.2. The global before-default optimization problem. The solution of the global before-default optimization problem is also obtained using another BSDE as in [4, Theorem 4.2]. We then get

$$
V_{C}(x)=U\left(x-Y_{0}\right)=-\exp \left(-p\left(x-Y_{0}\right)\right),
$$

where $\left(Y_{t}, Z_{t}\right)$ is a solution of the BSDE

$$
Y_{t}=C^{\mathbb{F}}+\frac{1}{p} \log G_{T}+\int_{t}^{T} f\left(s, Y_{s}, Z_{s}\right) d s-\int_{t}^{T} Z_{s} d W_{s},
$$

for $t \in[0, T]$ (note that (23) is exactly the equation $E_{0}$ in [4]), with the driver depending on $Y_{t}^{d}$ - the solution of the previous BSDE - as follows

$$
\begin{aligned}
f(t, y, z) & =-\frac{\mu_{t}^{\mathbb{F}}}{\sigma_{t}^{\mathbb{F}}} z-\frac{1}{2 p}\left(\frac{\mu_{t}^{\mathbb{F}}}{\sigma_{t}^{\mathbb{F}}}\right)^{2}+\inf _{v \in \mathscr{A}^{\mathbb{F}}}\left\{\frac{p}{2} \mid z+\right. \\
& \left.+\frac{1}{p} \frac{\mu_{t}^{\mathbb{F}}}{\sigma_{t}^{\mathbb{F}}}-\left.\sigma_{t}^{\mathbb{F}} v\right|^{2}-\frac{1}{p} U\left(y+v \gamma_{t}-Y_{t}^{d}(t)\right)\right\} .
\end{aligned}
$$

Finally, by equation (10), the utility indifference price can be calculated by

$$
\begin{aligned}
\operatorname{Pr}= & +\frac{1}{p} \log \left(-\sup _{\pi \in \mathscr{A}}\left(E^{\mathbb{P}^{*}}\left[U\left(X_{T}^{\pi}-B\right) \mid X_{0}^{\pi}=x\right]\right)\right)= \\
& +\frac{1}{p} \log \left(-\sup _{\pi \in \mathscr{A}}\left(E\left[U\left(X_{T}^{\pi}-C\right) \mid X_{0}^{\pi}=x\right]\right)\right)=Y_{0} .
\end{aligned}
$$

\section{Numerical results}

For illustration, we price an European call option written on a security exposed to a risk of counterparty default. The density of default time is assumed to be an exponential distribution with constant intensity $\lambda>0: \alpha(\theta)=\lambda e^{-\lambda \theta}$. This assumption implies the immersion property (see [1]) and consequently $W^{\mathbb{G}}=W$ and $J=0$. Moreover, we assume the size of the jump $\gamma$ is constant.

In the proof of Theorem 1 we have

$$
d L_{t}=L_{t^{-}}\left\{\beta_{t} \sigma_{t} d W_{t}^{\mathbb{G}}+\left(e^{\beta_{t} \gamma} e^{\phi_{t}}-1\right) d M_{t}\right\} .
$$

$L$ has the $\mathbb{G}$-decomposition of the form

$$
L_{t}=L_{t}^{\mathbb{F}} I_{\tau>t}+L^{d}(\tau)_{t} I_{\tau \leq t}, \quad 0 \leq t \leq T,
$$

where $L_{t}^{\mathbb{F}}$ and $L_{t}^{d}(\theta)$ are governed by, respectively,

$$
\begin{aligned}
d L_{t}^{\mathbb{F}} & =L_{t}^{\mathbb{F}}\left(\beta_{t}^{\mathbb{F}} \sigma_{t}^{\mathbb{F}} d W_{t}-\left(e^{\beta_{t}^{\mathbb{F}}} \gamma^{\phi_{t}^{\mathbb{F}}}-1\right) \lambda d t\right), 0 \leq t \leq T, \\
L_{0}^{\mathbb{F}} & =1,
\end{aligned}
$$

and

$$
\begin{aligned}
d L_{t}^{\mathbb{F}}(\theta) & =L_{t^{-}}^{d}(\theta)\left(\beta_{t}^{d}(\theta) \sigma_{t}^{d}(\theta) d W_{t}\right), \quad 0 \leq t \leq T, \\
L_{0}^{\mathbb{F}}(\theta) & =L_{\theta^{-}}^{\mathbb{F}}\left(e^{\beta_{\theta}^{\mathbb{F}} \gamma} e^{\phi_{\theta}^{\mathbb{F}}}\right) .
\end{aligned}
$$

Also by equation (15), $\beta_{t}$ could be decomposed as

where

$$
\beta_{t}=\beta_{t}^{\mathbb{F}} I_{\tau \geq t}+\beta_{t}^{d}(\tau) I_{\tau<t}
$$

$$
\beta_{t}^{d}(\theta)=-\frac{\mu_{t}^{d}(\theta)}{\left(\sigma_{t}^{d}(\theta)\right)^{2}}, \quad t \in[\theta, T],
$$

and $\beta_{t}^{\mathbb{F}}$ is solution of

$$
\mu_{t}^{\mathbb{F}}+\left(\sigma_{t}^{\mathbb{F}}\right)^{2} \beta_{t}^{\mathbb{F}}+\gamma \lambda e^{\phi_{t}^{\mathbb{F}}} e^{\beta_{t}^{\mathbb{F}} \gamma}=0, \quad t \in[\theta, T]
$$

where $\phi_{t}^{\mathbb{F}}$ solves the following ODE

$$
\begin{aligned}
\left(\phi_{t}^{\mathbb{F}}\right)^{\prime} & =F_{t}^{\mathbb{F}}(\beta, \phi)+\frac{d}{d t}\left(\int_{t}^{T} F_{s}^{d}(t)(\beta) d s\right), \quad t \in[\theta, T], \\
\phi_{T}^{\mathbb{F}} & =0 .
\end{aligned}
$$

Furthermore, $\beta_{t}^{\mathbb{F}}$ admits the following expression, using Lambert W-function (see [9])

$$
\beta_{t}^{\mathbb{F}}=-\frac{\mu_{t}^{\mathbb{F}}+\frac{\left(\sigma_{t}^{\mathbb{F}}\right)^{2} w_{t}}{\gamma}}{\left(\sigma_{t}^{\mathbb{F}}\right)^{2}}, \quad t \in[\theta, T]
$$


where

$w_{t}=$ lambertW $\left(\frac{\gamma^{2} \lambda \exp \left(\phi_{t}^{\mathbb{F}}\right) \exp \left(\frac{-\gamma \mu_{t}^{\mathbb{F}}}{\left(\sigma_{t}^{\mathbb{F}}\right)^{2}}\right)}{\left(\sigma_{t}^{\mathbb{F}}\right)^{2}}\right), t \in[\theta, T]$, and we have $w_{t} \in \mathbb{R}$ because $\frac{\gamma^{2} \lambda \exp \left(\phi_{t}^{\mathbb{F}}\right) \exp \left(\frac{-\gamma \mu_{t}^{\mathbb{F}}}{\left(\sigma_{t}^{\mathbb{F}}\right)^{2}}\right)}{\left(\sigma_{t}^{\mathbb{F}}\right)^{2}}>0>-\frac{1}{e}$. We suppose that $\mu^{\mathbb{F}}$ and $\sigma^{\mathbb{F}}$ are constant and $\sigma_{t}^{d}(\theta)$ is a deterministic function. In this example, we consider volatility after default $\sigma^{d}$ in two cases:

- We expect that the volatility $\sigma^{d}(\theta)$ after default is greater than the volatility $\sigma^{\mathbb{F}}$ before default, and the earlier the default takes place, the larger this gap becomes, for instance, $\sigma^{d}(\theta)=\sigma^{\mathbb{F}}\left(2-\frac{\theta}{T}\right)$.

- We also expect that the volatility $\sigma^{d}(\theta)$ after default is greater than the volatility $\sigma^{\mathbb{F}}$ before default. However, after the instantaneous increase, we suppose that the volatility will decays overtime to the before-default value (Fig. 1). For instance, we consider $\sigma_{t}^{d}(\theta)$ of the following form $\sigma_{t}^{d}(\theta)=\sigma^{\mathbb{F}}+k_{1} e^{-k_{2}(t-\theta)}$.

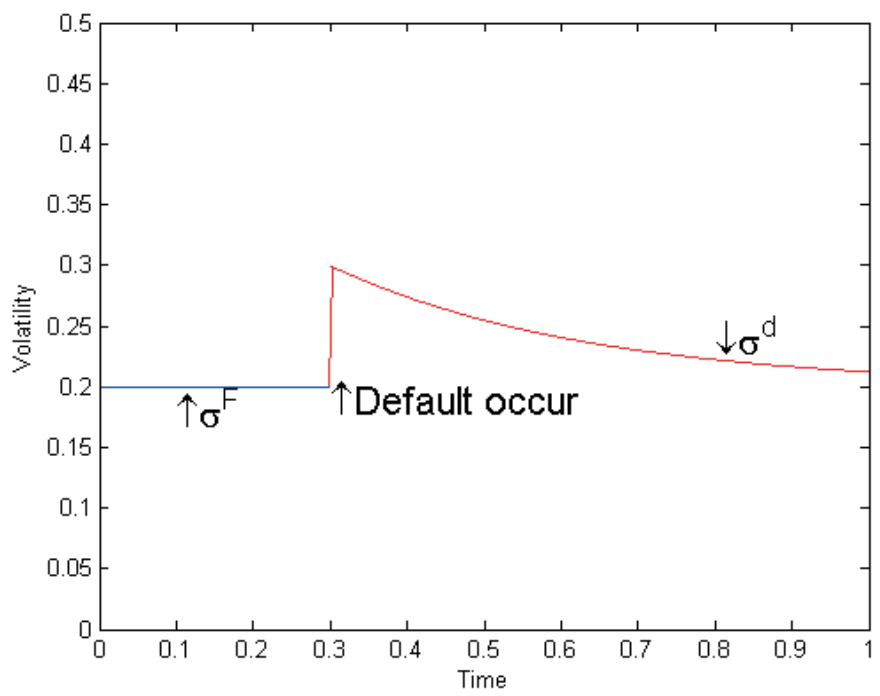

Fig. 1. For example: $\sigma^{\mathbb{F}}=0.2, \sigma_{t}^{d}(\theta)=\sigma^{\mathbb{F}}+0.1 e^{-3(t-\theta)}$ and $\tau=0.3$

We use a regression based method (see [10]) to simulate the above BSDE processes to price the option with parameters in Table 1.

Table 1

Results of parameters estimation

\begin{tabular}{|c|c|c|c|c|c|c|c|}
\hline$S$ & $K$ & $r$ & $\mu^{\mathbb{F}}=\mu^{d}$ & $\sigma^{\mathbb{F}}$ & $T$ & $\lambda$ & $p$ \\
\hline 100 & 100 & 0.05 & 0.05 & 0.2 & 1 & 0.1 & 1 \\
\hline
\end{tabular}

In Fig. 2, we present the dependence of indifference price on jump's size, classifying by three forms of volatility. We found that in the case where volatility is unchanged ("Indifference price 1" curve), the utility indifference price without jump (that is, jump's size is 0 ) is equal to the Black-Scholes

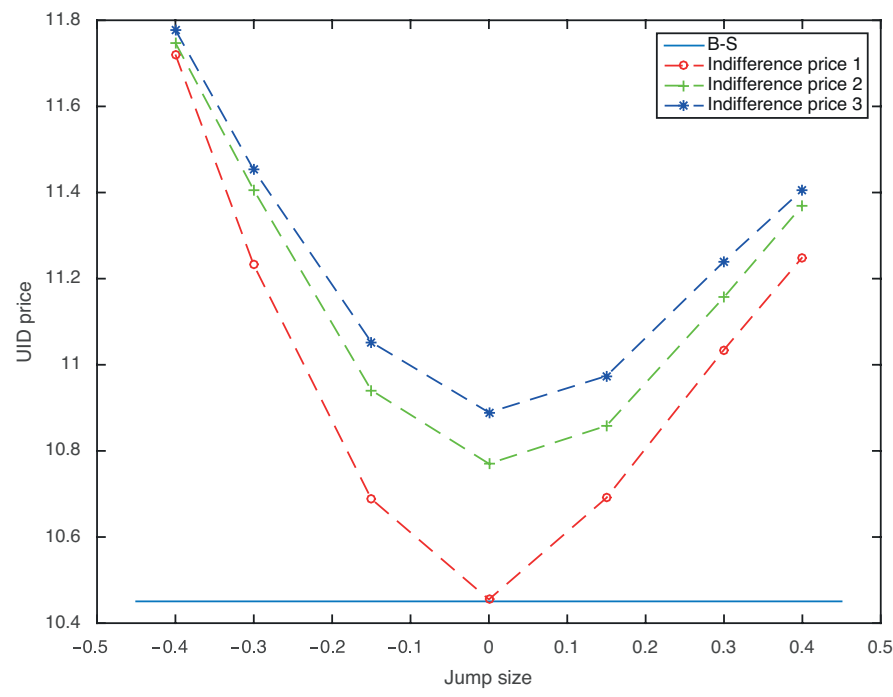

Fig. 2. "Indifference price 1": The volatility remains unchanged after default. "Indifference price 2": The relation between before-default volatility and after-default volatility is $\sigma^{d}(\theta)=\sigma^{\mathbb{F}}\left(2-\frac{\theta}{T}\right.$. "Indifference price 3": The relation between before-default volatility and after-default volatility is $\sigma_{t}^{d}(\theta)=\sigma^{\mathbb{F}}+0.2 e^{-0.1(t-\theta)}$

price of 10.4506 as expected. Furthermore, the indifference price is larger as the volatility after default is larger. In Fig. 3, we present the dependence of indifference price on the size of the jump and intensity of default in the case the volatility after default is unchanged.

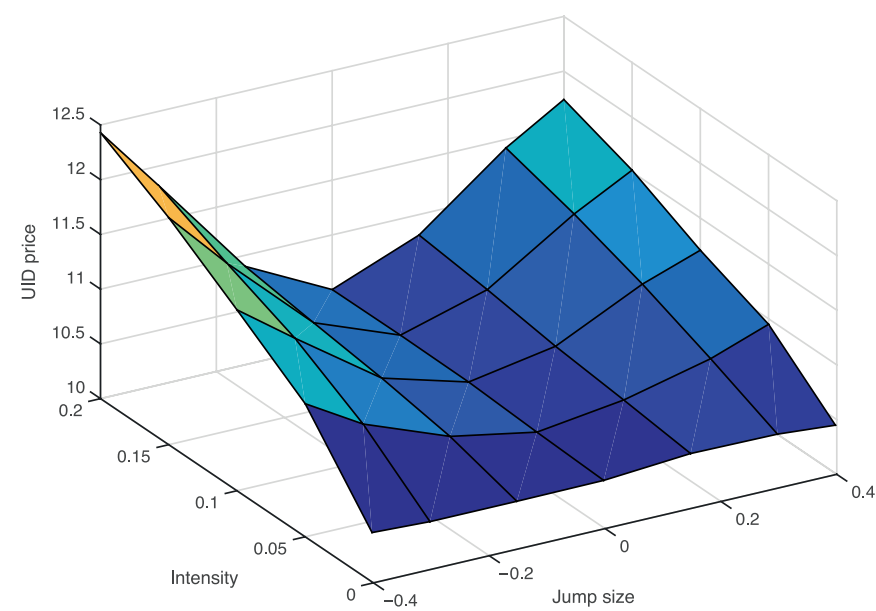

Fig. 3. The changing of price by intensity and jump size

\section{Conclusion}

This paper studies the valuation problem of a derivative in the presence of counterparty risk for the trading underlying asset, where the price, drift and volatility of the asset may change abruptly. We use the minimal entropy martingale measure approach to solve the utility indifference equation. This approach, 
combined with an exponential utility function, helps reduce the problem to solving a unique optimization problem. The main contribution of this work is the derivation of the MEMM density in the above framework (with the presence of counterparty risk). In order to solve the remaining optimization problem and derive the derivative's price, we employ the decomposition approach proposed by [4], and find the value function after and before the default successively. Finally, we demonstrate numerical calculation for a standard European option and are able to quantify the impact of the default (size of the jump, change in volatility) and its intensity on the derivative's price. This result is encouraging given the increasing awareness of counterparty risk in the financial market.

\section{REFERENCES}

[1] N. El Karoui, M. Jeanblanc, and Y. Jiao, "What happens after a default: the conditional density approach", Stoch. Proc. Appl. 120 (7), 1011-1032 (2010).

[2] S. D. Hodges, "Optimal replication of contingent claims under transaction costs", Review of Futures Markets 8, 223-238 (1989).
[3] M. Mania and M. Schweizer, "Dynamic exponential utility indifference valuation”, Ann. Appl. Probab. 15 (3), 2113-2143 (2005).

[4] Y. Jiao, I. Kharroubi, and H. Pham, "Optimal investment under multiple defaults risk: a BSDE-decomposition approach", Ann. Appl. Probab. 23 (2), 455-491 (2013).

[5] M. Jeanblanc and Y. Le Cam, "Progressive enlargement of filtrations with initial times", Stoch. Proc. Appl. 119 (8), 2523-2543 (2009).

[6] R. Mansuy and M. Yor, Random Times and Enlargements of Filtrations in a Brownian Setting, Springer, 2006.

[7] P. Protter and K. Shimbo, "No arbitrage and general semimartingales" in Markov Processes and Related Topics: a Festschrift for Thomas G. Kurtz, 267-283, Institute of Mathematical Statistics, 2008.

[8] S. Ihara, Information Theory for Continuous Systems, vol. 2, World Scientific, 1993.

[9] R.M. Corless, G.H. Gonnet, D.E. Hare, D.J. Jeffrey, and D.E. Knuth, "On the Lambert w function", Adv. Comput. Math. 5 (1), 329-359 (1996).

[10] E. Gobet, J.-P. Lemor, and X.Warin, "A regression-based Monte Carlo method to solve backward stochastic differential equations", Ann. Appl. Probab. 15 (3), 2172-2202 (2005). 